\title{
Sulfur cycle dynamics during the Great Oxidation Event
}

\author{
KAZUMI OZAKI $^{1}$, YUNA MIURA ${ }^{1}$ \\ ${ }^{1}$ Department of Environmental Science, Toho University, \\ Funabashi, Chiba 274-8510, Japan \\ [kazumi.ozaki@sci.toho-u.ac.jp]
}

During the early Paleoproterozoic, the Earth system has seen a series of environmental changes, including severe glaciations and a dramatic increase in atmospheric oxygen $\left(\mathrm{O}_{2}\right)$ levels (Great Oxidation Event; GOE). The oxygenation of Earth surface environment would be a primary driver of biological evolution by reshaping the landscape of biogeochemical cycles. The recent constraint on the sulfur isotopic records in the aftermath of the third glaciation (Gowganda/Rooihoogte Formations) implies an intimate link between the glaciation and the GOE $(2.33 \mathrm{Ga})$. However, the biogeochemical dynamics throughout the GOE is still puzzling.

Here we use a numerical model of the global biogeochemical cycles of carbon, phosphorus, sulfur and oxyen to examine the mechanistic explanation for the large negative excursion of sulfur isotope of pyrite $\left(\delta^{34} S_{p y}\right)$ shortly after the GOE. We paid a special attention to the sulfur cycle dynamics because sulfur isotope data provides powerful quantitative constraints on the redox-driven biogeochemical cycles in Earth surface environment. We explore the impact of varying key model parameters using a stochastic approach and find that the extremely high atmospheric $\mathrm{CO}_{2}$ levels $(>0.2 \mathrm{~atm})$ would be required as an initial condition (in the afatermath of the glaciation) to cause a sufficient negative anomaly within 10-20 Myr after the glaciation. This implies that the third glaciacion was a snowball earth event. Our model also provides a series of testable predictions, including a secular positive excursion of $\delta^{34} \mathrm{~S}$ of both seawater sulfate $(+20-30$ permil) and pyrite $(+0-5$ permil) and a buildup of seawater sulfate to $>1 \mathrm{mM}$ on the timescale of $10^{7}$ years. These model predictions should be tested by the geologic records. 